\title{
On a Simple Instrument, Siomi-ito, for Detecting Underwater Current.
}

\author{
Tasae KawaKami and Yùnosuke IITAKa \\ (Fisheries Dept., Kyôto Univ., Maizuru)
}

Siomi-ito, which is a simple device for detecting underwater currents, is locally in use among Japanese fishermen in coastal fishing grounds, espacially in the case when they are going to set their encircling net. When such a net is laid out in a water where there exists a strong underwater current relative to the surface one, it happens not seldom to occur that the net is entangled itself by the disturbed current, thus a part of the bottom margin of the net is caught in the purse line and pulled into a purse ring with the purse line*. When it goes wrong, there is no help to mend matters. Fishermen, therefore, are in a great care in finding out the underwater strong current before they shoot their net. In such a case, a Siomi-ito is tried. to use by the chief of the crew on board the skiff. This consists of dropping several boulder weights attached to strings of different lengths. Each weight is subjected to the current of the corresponding depth according to the length of string. The inclination of the string at the surface of the sea will be determined by both the profile of the current velocity and the length of the string. A skillful fisherman can $t: 11$ the particularly unfavourable violent underwater current by a glance of the inclination angles of the strings. The judgement is said to be hardly possible only by experienced fishermen. It is said that they judge erroneously at times and the crew suffers from getting out of the trouble.

Although the detection of underwater current is an urgent problem in fishing operations, the suitable device for fishery purpose has not yet been investigated scientifically. The difficulty seems to arise from the fact that the current velocity at each depth has to be measured successively as a vector quantity, even if it is a rough estimation. Judging from the fisheries point of view, on account of its simplicity, the Siomi-ito may be a hopeful gear for haul seine fishery of a small scale, operating in the water where there is some possibility of occurence of predominant underwater current.

Siomi-ito's which are now in common use in Japan are made at fisherman's own way; each skipper has his own idea and one should not expect to find two Siomi-ito's just alike. The authors were intended to make clear the theory of Siomi-ito and to study the proper way of its make up and manipulation for fishery use.

\section{Theoretical Considerations}

It has been well established by many authorities that the normal component of the hydrodynamic force acting upon the string subjected to a uniform current varies proportionally with the square of the sine of the angle between string and the stream and that its tangential component may be negligible as compared with the normal component especially when the angle is not so far apart from $90^{\circ}$.

Suppose a spherical sinker which is lowered into the water, where the distribution of current, $U$, is homogeneous and horizontal, by a flexible fine string as shown in

Received September 24, 1954.

* This fhenomenon is frequently called "Kankui" by Japanese fishermen. 
annexed figure. In a system of rectangular coordinates $(x, z)$, whose origin, $O$, is located at a point on the string at the water surface, let the $z$-axis be directed verti-

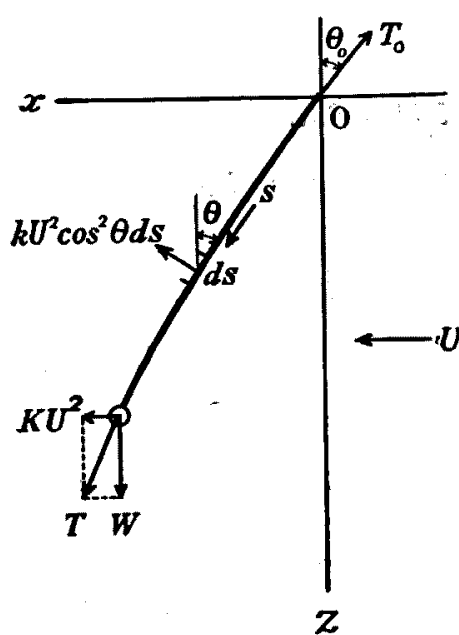
cally downwards and let the $x$-axis be parallel to the stream line, measured positive downstream. Let $s$ be the arc length along the string from the origin to any point $\mathrm{P}(\boldsymbol{x}, z)$ on the string, and let $\theta$ be the inclination angle i.e., the angle between the tangent of the string and the $z$-axis at this point. If the weight of the string be compensated by the buoyancy, and the tangential force acting along it be negligible, its tension, $T$, may be constant throughout the whole string. Then the equation of equiribrium may be written as

$$
-T \frac{d \theta}{d s}=R \sin ^{2}\left(\frac{\pi}{2}-\theta\right)=R \cos ^{2} \theta,
$$

where $R$ is the drag of the string of unit length when it is placed perpendicularly to the current, i.e.,

$$
R=k U^{2} \text {, }
$$

$k$ being a constant depending only upon the size of the string, provided that NewToN's law for hydrodynamic drag is tenable. The integration of this differential equation, with the condition that $\theta=\theta_{0}$ for $s=o$, gvies

$$
\frac{R}{T} s=\tan \theta_{0}-\tan \theta,
$$

and

$$
\frac{R}{T} z=\log \tan \left(\frac{\pi}{4}+\frac{\theta_{0}}{2}\right)-\log \tan \left(\frac{\pi}{4}+\frac{\theta}{2}\right) .
$$

For the case in which the inclination angle $\theta$ is small, the expressions can be simplified to

$$
\frac{R}{T}-s=\frac{R}{T} z=\theta_{0}-\theta
$$

Next, suppose the water in which a definite but unknown velocity distribution along the $z$-axis may exist. Then devide the water into several thin horizontal sublayers of constant thickness $L$ so that the current can be assumed to be homogeneous throughout each layer. Assign the number $1,2, \cdots, n$ to these layers from the surface toward the bottom. Let $U_{i}$ be the current velocity at the $i$-th layer. Next, when $n$ spherical sinkers above mentioned are suspended simultaneously by $\boldsymbol{n}$ strings so as to locate at the center of each layer, denote by $\theta_{i}, o$ the inclination angle of the string which carries the sinker being suspended at the $i$-th layer, at the water surface and denote by $\theta_{\ell}$ the inclination angle of the string at the joint of the $i$-th sinker. If NEwTon's law be tenable for the drag of the sinker, we have

$$
\theta_{i} \risingdotseq \tan \theta_{i}=\frac{K}{W} U_{i}^{2} \risingdotseq \frac{K^{r}}{T} U_{i}^{2},
$$

where $K$ is the proportionality constant depending upon the projected area of the sphere and $W$ its apparent weight, which is equal, without sensible inaccuracy, to the tension, $T$, of the string if $\theta$ be small.

Applying the equations (2), (3) and (4) to the first string, we have 


$$
\frac{1}{T}\left(\frac{k L}{2}+K\right) U_{1}^{2}=\theta_{1,0}
$$

For the $i$-th string, similarly we have

$$
\frac{k L}{T}\left(U_{1}^{2}+U_{2}^{2}+\cdots+U_{i-1}^{2}\right)+\frac{1}{T}\left(\frac{k L}{2}+K\right) U_{i}^{2}=\theta_{i}, 0 .
$$

Then subtracting the equation for the $i$-th string from that for the $(i+1)$-th string, a reduction formula for $U$ can be obtained as follows :

$$
\frac{1}{T}\left(\frac{k L}{2}+K\right) U^{2_{i+1}}+\frac{1}{T}\left(\frac{k L}{2}-K\right) U_{i}^{2}=\theta_{i+1,0}-\theta_{i, 0} \equiv \Delta \theta_{i},
$$

by means of which, together with the equation ( 5 ), the values of $U_{i}$ may be roughly estimated layer by layer by measuring the differences of inclination angles, $\Delta \theta_{i}$, of successive strings at the surface.

If all the strings are not laid on one plane, it is natural to treat all values by decomposing them into two perpendicularly intercepted directions.

\section{Some Remarks on the Design of Siomi-ito}

In order to save the trouble in computation, some expedients on the design of Siomi-ito are advisable. Let us consider the case in which

$$
L=\frac{2 K}{k} \text {. }
$$

Then the equations (5) and (6) are simplified to

$$
\stackrel{2}{T} U_{i+1}^{*}=\Delta \theta_{i}
$$

by means of which the computation may be much simplified. In these equations, since the values of $K$ and $k$ are given by ordinary hydrodynamical considerations, the length $L$ may be determined by the diameters of the string and the spherical sinker used, and $T$ by the density of the sinker. Thus we may be able to design a Siomi-ito. which meets our requirment.

As regards the string, it must be kept in mind that the roughness of the surfaceof strings should be smoothed by coating any material such as plastic film or a braid. of circular cross section should be used, so that the string may be free from the yawing" action which may arise by the helicoidal pattern of the strand. As to the material of string, a fiber of the lowest density possible should be employed in order to balance its buoyancy. Nylon cord would be recommended from this point of view.

The expenses for executing this study were partly defrayed by the research fund from the Japanese Ministry of Education.

\section{Reference}

1). PODE, L ; An experimental investigation of the hydrodynamic forces on stranded cables. Report of the David W. Taylor Model Basin (U.S. Navy Dept.), No. 713, 1950. 